\title{
Manufacturing Process Technology of Dual-wavelength Laser Direct-write Nanolithography System for Gold-nanoparticle-based Localized Surface Plasma Resonance Biosensor
}

\author{
Hsing-Cheng Yu, ${ }^{*}$ Shuen-Chen Chen, ${ }^{2}$ and Ding-Zheng Lin $^{3}$ \\ ${ }^{1}$ Department of Systems Engineering and Naval Architecture, National Taiwan Ocean University, \\ 2 Pei-Ning Road, Keelung 20224, Taiwan \\ ${ }^{2}$ Biomedical Technology and Device Research Laboratories, Industrial Technology Research Institute, \\ 195, Sec. 4, Chung Hsing Road, Chutung, Hsinchu 31040, Taiwan \\ ${ }^{3}$ Material and Chemical Research Laboratories, Industrial Technology Research Institute, \\ 195, Sec. 4, Chung Hsing Road, Chutung, Hsinchu 31040, Taiwan
}

(Received July 11, 2017; accepted November 21, 2017)

Keywords: biosensor, dual wavelength, localized surface plasma resonance, nanolithography

Recently, in vitro diagnostics (IVD) has been widely applied in the qualitative and quantitative analyses of medical diagnoses and drug development in biomedical technology. There are $\sim 50 \%$ global IVD markets including immunoassay, molecular diagnostics, point-ofcare testing, and biosensor detection. Therefore, the development of a manufacturing process technology for biosensor detection that is cost-effective and highly sensitive is important. In general, the biochip development of metal nanostructure arrays adopting localized surface plasma resonance (LSPR) is based on mechanical nanolithography with a tip of an atomic force microscope. However, it does not improve the irregular shapes and dimensions during the mass production of biochips, and does not increase the process area, so sensitivity is sacrificed. In this paper, we present a novel dual-wavelength laser direct-write nanolithography system for fabricating periodic gold nanoparticle arrays on substrates for biosensors. The substrates were immersed by a wet etching method after exposure. Isotropic etching using oxygen-plasma method transfer to the substrates was then performed to fabricate a suitable undercut in the liftoff of the dry etching process. The pitch, depth, and width of the periodic gold nanoparticle arrays were 500, 43, and $197 \mathrm{~nm}$, respectively, and the measured transmission spectrum was $800 \mathrm{~nm}$. Chips based on the periodic gold nanoparticle arrays were designed for LSPR biosensors with high sensitivity, to be widely applied in biomedical fields.

\section{Introduction}

With the advancement of biomedical technology in recent years, in vitro diagnostics (IVD) has been widely applied to the qualitative and quantitative analyses of medical diagnoses and drug development via rapid development in biomedical engineering. Immunoassay, molecular diagnostics, and point-of-care diagnostics comprise $\sim 50 \%$ of the global IVD market, and

*Corresponding author: e-mail: hcyu@ntou.edu.tw

http://dx.doi.org/10.18494/SAM.2018.1754 
biosensor detection is one of the main products. Therefore, it is an important research topic to develop a cost effective and highly sensitive biosensor detection technology. A chip based on the localized surface plasmon resonance (LSPR) technique is excited by metallic nanostructures, which is different from Kretschmann configuration (i.e., prism coupling) of conventional surface plasmon resonance (SPR). Nanostructure LSPR arrays can be fabricated using different methods, such as electron beam and nanosphere lithographies. ${ }^{(1,2)}$ Biosensor development of metal nanostructure arrays is generally based on mechanical nanolithography with the tip of an atomic force microscope (AFM). However, the irregular shapes and dimensions were not suitable for the fabrication of biosensors. Moreover, the fabricated area was limited, thus the detection sensitivity was sacrificed. In the last decade, many advanced research laboratories have demonstrated biosensor detection techniques using LSPR biosensors. ${ }^{(3)}$ Additionally, the main operating principle was to use a full band of light to excite the micro-/nano-dimensional metal particles to obtain LSPR, and the localized electromagnetic field is generated on the metal particles or structures. ${ }^{(4)}$ Zou et al. presented an arrangement method using a periodic nanoparticle cycle effect to narrow the LSPR spectrum of nanoparticles and to obtain a high sensitivity. ${ }^{(5)}$ Using the same concept, a metal nanoparticle was proposed to maintain their periodic arrangement in obtaining LSPR with a narrow bandwidth, and it has been widely adopted in the construction of nanostructures. ${ }^{(6)}$ Although many designs of successful LSPR biosensors already exist, there are still many opportunities to improve their performance and applications. The spectral shift of the adsorbed amount of molecules is guaranteed by the LSPR biosensor because the resonance wavelength of the LSPR sensor is sensitive to the change in the local refractive index near the metal nanostructures. As a consequence, the optomechanical platform of the LSPR biosensors can be prepared in a simpler and more compact way than that of the platform of the SPR biosensors. ${ }^{(7)}$ An optomechanical platform is presented in this study to achieve the system characteristics with high sensitivity and is superior to the conventional SPR in biomedical detection, and is well suited to the quick, sensitive, label-free, and multiplexed detection of molecules. Therefore, in this paper, we present a novel miniaturized dual-wavelength laser direct-write nanolithography system (DLDNS) that consists of an optical pickup head (OPH) system with dual-path optical design, a servo control system with a laser diode drive controller, an autofocus (AF) actuator with AF function, and a positioning stage system composed of a linear moving stage and a spindle motor. Moreover, the system integration of the stable exposure output of the blue-violet laser, accurate AF function of the red laser, and positioning servo control of the nano scale linear moving stage minimized the dimensional errors of the nanostructure arrays and reduced the structural dimension effectively. The heat-mode inorganic photoresist films were exposed by the blue-violet laser source in the DLDNS, and the improved optical diffraction limited resolution enabled the fabrication of the periodic subwavelength nanoparticle arrays on the substrate surfaces. The substrate surfaces were immersed by the wet etching method after the exposure. Isotropic etching using the oxygen-plasma method transferred the pattern to the substrates was then used to fabricate a suitable undercut in the lift-off of the dry etching process. The LSPR biochips based on the developed periodic gold nanoparticle arrays were fabricated using a thermal directwrite nanolithography process. The optimization of the periodic gold nanoparticle arrays 
enabled better spectrum response and stronger localized field, which improve the sensitivity of biosensor chips. As a consequence, the DLDNS was used to efficiently fabricate the LSPR biosensors with periodic arrays of nanostructures. In the manufacturing process the following characteristics were produced: high throughput (large area and quick production), costeffectiveness, and increased detection sensitivity.

\section{Materials and Methods}

\subsection{Design of OPH in DLDNS}

An OPH with dual-path optical design in the DLDNS and its photograph are shown in Figs. 1(a) and 1(b), respectively. There were two light rays for the laser sources with dualwavelengths in this study; the first was a blue-violet laser with a wavelength of $405 \mathrm{~nm}$ on the left-hand side, and the second was a red laser with a wavelength of $658 \mathrm{~nm}$ on the right-hand side. The blue-violet laser with the shorter wavelength was adopted as a light source to fabricate the periodic nanostructure arrays. Moreover, the red laser was applied to the AF function to maintain a fixed operational distance in the DLDNS. For both light rays, we adopted quadrant detectors to detect generated focus error signals using an astigmatic focusing detection method. According to the forward light path, both the blue-violet and red light rays were designed as directional lights. A branch of the blue-violet light ray entered a collimator \#1, a polarizing beam splitter (PBS) \#1 of the transmitted P-polarization, a beam splitter (BS) \#1, a fold mirror with a $45^{\circ}$ angle of inclination, a quarter wave plate (QWP), and the objective with a 0.85 numerical aperture to focus on the sample. Moreover, another branch of the blue-violet light was reflected by the fold mirror to enter the PBS \#1 of the reflected S-polarization, a detector lens \#1, and a cylinder lens, before reaching the photodetector integrated circuit (PDIC) \#1. The red light ray entered the PBS \#2, a collimator \#2, the BS \#1, the fold mirror, the QWP, and the objective to focus on the same sample. Both light rays in the DLDNS merged at the BS \#1 to reach the objective. In addition, light was reflected into the feedback path through the objective, the QWP, and the fold mirror, to reach the BS \#1. A branch of the blue-violet feedback light

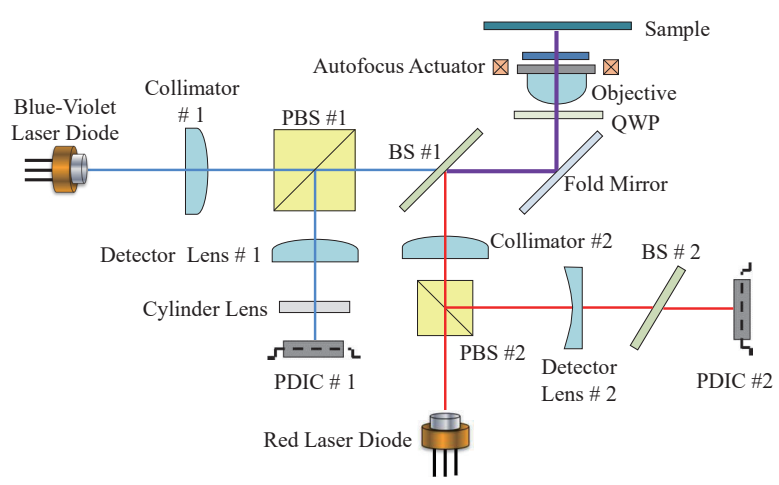

(a)

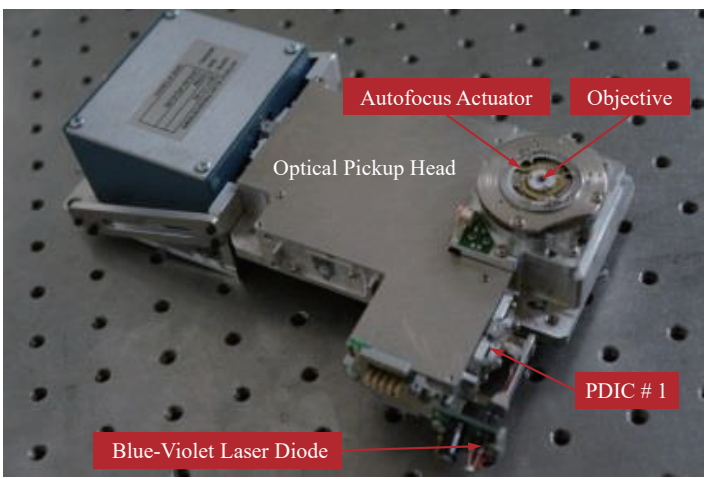

(b)

Fig. 1. (Color online) (a) An OPH with dual-path optical design in the DLDNS and (b) a photograph of the OPH. 
ray from the BS \#1 entered the PBS \#1, the detector lens \#1, and the cylinder lens, to reach the PDIC \#1. Furthermore, a branch of the red feedback light ray from the BS \#1 passed through the collimator \#2, the PBS \#2, a detector lens \#2, and a BS \#2 to reach the PDIC \#2.

In general, the accepted definition of the spot size $d_{F W H M}$, for the full width at half maximum (FWHM), on a focal plane in an optical system is related to its wavelength and NA by

$$
d_{F W H M}=0.61 \frac{\lambda}{N A},
$$

where $\lambda$ is the wavelength, and $N A$ is the numerical aperture of the objective in the optical system that is given by

$$
N A=n \times \sin \theta \cong n \times \tan \theta\left(\text { if } \theta \ll 1^{\circ}\right) \cong \frac{n \cdot D}{2 f} .
$$

Here, $n$ is the refractive index, $\theta$ is the angle between the converging light and the optical axis, $D$ is the effective aperture of the objective, and $f$ is the focal length of the objective. The laser light passes through the objective, i.e., an AF lens, to converge to the laser beam, and the smallest spot position defines the best focus. If the placement tolerance of the laser focal point is insignificant, the tolerance range $\Delta Z$ is the depth of focus, and is given by

$$
\Delta Z=0.6 \frac{\lambda}{(N A)^{2}}
$$

However, the depth of focus is highly sensitive to the $N A$ of the objective. By using a high- $N A$ lens, the spot size can be reduced, and the focus accuracy increases with an AF controller.

\subsection{Experimental operation of DLDNS}

The experimental block and device diagrams of the DLDNS are illustrated in Figs. 2(a) and 2(b), respectively. The compact DLDNS consisted of an OPH system with a dual-path optical design, a control system with a laser diode drive controller, an AF controller, and motor and stage controllers, as well as a stage system composed of a spindle motor and a linear moving stage. The laser diode drive controller can be adjusted using the pulse-width modulation (PWM) signals, and the blue-violet laser is operated at a $405 \mathrm{~nm}$ wavelength. The NA of the objective was 0.85 . In addition, the AF servo controller, spindle motor, and stage drive controllers in the control system were used in a closed-loop using a personal computer for the rotational and linear movements. Therefore, the laser beam focused on the sample, i.e., a glass substrate, deposited with a heat-mode inorganic photoresist film of $65 \mathrm{~nm}$ thickness. The DLDNS was used to efficiently fabricate the LSPR biosensors to achieve a cost-effective manufacturing process with high-throughput (large area and quick production). As a consequence, the developed DLDNS in this study was used to overcome the resolution limit of optical diffraction and had a smaller spot size suitable for fabricating LSPR biosensors with periodic arrays of nanostructures. 


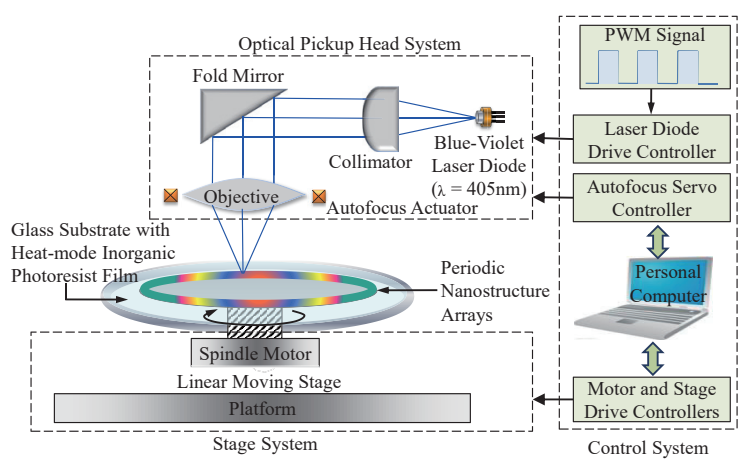

(a)

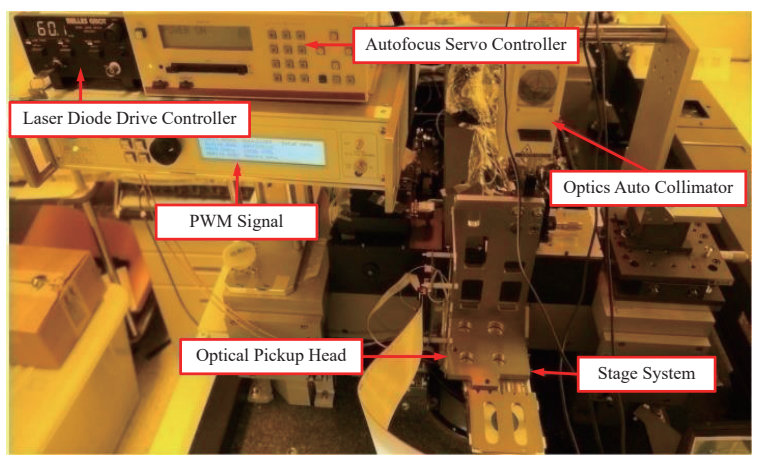

(b)

Fig. 2. (Color online) Experimental (a) block diagram and (b) device diagram of the DLDNS.

The detection sensitivity of the LSPR biosensors with periodic arrays of nanostructures was improved.

\subsection{Optical properties and principles of gold nanoparticle arrays}

The optical properties of gold nanoparticle arrays are significantly different from those observed in the bulk material. In LSPR, when light is incident on the gold nanoparticle arrays, the light of a specific frequency can interact with the gold nanoparticle arrays and induce coherent oscillations of the conduction electrons on the gold surface. For a sample with spherical metal nanoparticles in a homogeneous medium, the extinction spectra $C_{\text {ext }}$ can be derived from the Mie theory and the Drude model. ${ }^{(8,9)}$

$$
C_{e x t}=\frac{24 \pi^{2} r^{3} \varepsilon_{m}^{2 / 3}}{\lambda} \times \frac{\varepsilon^{\prime \prime}}{\left(\varepsilon^{\prime}+2 \varepsilon_{m}\right)^{2}+\varepsilon^{\prime 2}}
$$

Here, $r$ is the radius of the nanoparticle, $\varepsilon_{m}$ is the dielectric constant of the surrounding medium, and $\varepsilon=\varepsilon^{\prime}+i \varepsilon^{\prime \prime}$ is the complex dielectric constant of the bulk metal. According to Eq. (4), the maximum extinction occurs when $\varepsilon^{\prime \prime}$ is relatively small and $\left(\varepsilon^{\prime}+2 \varepsilon_{m}\right)$ approaches zero. For certain metals, e.g., gold or silver, if the incident light is in the visible wavelength range, this relationship is satisfied, where $\varepsilon^{\prime \prime}$ is small and $\varepsilon^{\prime}$ is negative. Moreover, the real part of the dielectric constant of gold, $\varepsilon^{\prime}$, is strongly dependent on wavelength, leading to a shift of the resonance wavelength when there is a refractive index change in the surrounding medium, i.e., $\varepsilon_{m}$. This phenomenon illustrates why periodic arrays of gold nanoparticles are the most widely used candidates for refractive index sensors. However, for a complex nanostructure fabricated on the glass substrate (such as LSPR biosensors), there is no simple analytical solution.

The resonance wavelengths of LSPR biosensors in different surrounding media were calculated using the finite difference time domain (FDTD) simulation method. In the 
simulation, the width and depth of the periodic gold nanoparticle arrays were 200 and $40 \mathrm{~nm}$, respectively, and the thickness of the adhesive chrome layer was $3 \mathrm{~nm}$. The refractive index of the glass substrate was 1.58 and the refractive index of the surrounding medium was in the range from 1.0 to 1.5. The arrangement of the periodic gold nanoparticle arrays was made of rectangular arrays with a pitch of $500 \mathrm{~nm}$. The characteristic wavelength of the LSPR spectral shift, $\Delta \lambda$, is approximately described as $(10,11)$

$$
\Delta \lambda \approx m(\Delta n)\left(1-e^{-\frac{2 d}{l_{d}}}\right)
$$

where $m$ is the bulk sensitivity of the LSPR biosensor in units of nanometers per refractive index $(\mathrm{nm} / \mathrm{RIU}), \Delta n$ is the refractive index changes of the surrounding medium, $d$ is the effective thickness of the adsorbed layer, and $l_{d}$ is the decay length of the electromagnetic field. To estimate the bulk sensitivity and measure the spectral changes, different oils with standard refractive indices were added to the surrounding media of the nanostructures. In this study, the thickness of the adsorbed layer was much greater than the decay length of the electromagnetic field $\left(d \gg l_{d}\right)$, and Eq. (5) can be simplified as

$$
\Delta \lambda \approx m \Delta n .
$$

\section{Experimental Results and Discussion}

In the exposure experiment, organic photoresist and heat-mode inorganic photoresist films were coated on 4-inch-diameter glass substrates. The blue-violet laser with a $405 \mathrm{~nm}$ wavelength and the objective with $0.85 \mathrm{NA}$ were used in the DLDNS. Moreover, the AF and exposure using red and blue-violet lasers, respectively, produced a confocal energy distribution on the glass substrate. Therefore, the structural uniformity was directly affected by the absorbed heat upon the heat-mode inorganic photoresist material. Furthermore, various nanostructure arrays considerably affected the sensitivity of the LSPR biosensors due to the exposure energy and matching power. The laser diode drive controller was adjusted by the optimal PWM signal, and the DLDNS overcame the resolution limit of the optical diffraction with a smaller spot size from the laser beam focused on the heat-mode inorganic photoresist films deposited on the glass substrates. Therefore, the LSPR biosensors with periodic nanostructure arrays were fabricated and exposed on the heat-mode inorganic photoresist films by the novel DLDNS in this study. The exposed inorganic photoresist films on the glass substrates could be etched away in the manufacturing process. To prevent the unexposed inorganic photoresist films from etching in potassium hydroxide $(\mathrm{KOH})$ and to obtain higher depth-to-width ratios of the nanostructures, the optimal exposure energy and etching time must be precisely controlled in the DLDNS. The films were immersed in an alkaline solution of $0.05 \mathrm{M} \mathrm{KOH}$ for $100 \mathrm{~s}$ by the wet etching method after exposing with the laser direct-write process, before being rinsed with deionized water. The substrate surfaces were then dried with nitrogen. Next, the oxygen flow rate of the oxygenplasma at low temperature had to be modified, so that the isotropic dry etching process could be transferred to the organic photoresist films to fabricate a suitable undercut for the following 
lift-off process. Also, to facilitate the lift-off process, the bottom of the organic photoresist was removed due to the undercut shape. The process parameters of the oxygen plasma were a flow rate of $200 \mathrm{ml} / \mathrm{min}$, power of $800 \mathrm{~W}$, etching time of $5 \mathrm{~min}$, and 5 cycles. The gold targets were finally deposited on the films using the thermal evaporation method and then the periodic arrays of gold-nanoparticles were produced by acetone in the lift-off process. An optical microscope and AFM topographical scan of the nanostructures are shown in Figs. 3(a) and 3(b), respectively. The dimensional features of the periodic gold nanoparticle arrays on the substrate surfaces of the LSPR biosensors can be observed; the width, depth, and pitch were 197, 43, and $500 \mathrm{~nm}$, respectively, and the measured transmission spectrum was $800 \mathrm{~nm}$. The transmission spectrum of the gold-nanoparticle-based biosensors in the FDTD simulation is shown in Fig. 4(a), and the bulk sensitivity of the gold-nanoparticle-based biosensors in experiments was $\sim 170$ $\mathrm{nm} / \mathrm{RIU}$, calculated from the slope of the characteristic peak of the refractive index changes derived from Fig. 4(b). The FDTD simulation results agree with the measured transmission spectrum of the LSPR biosensors for different refractive indexes in Fig. 5.

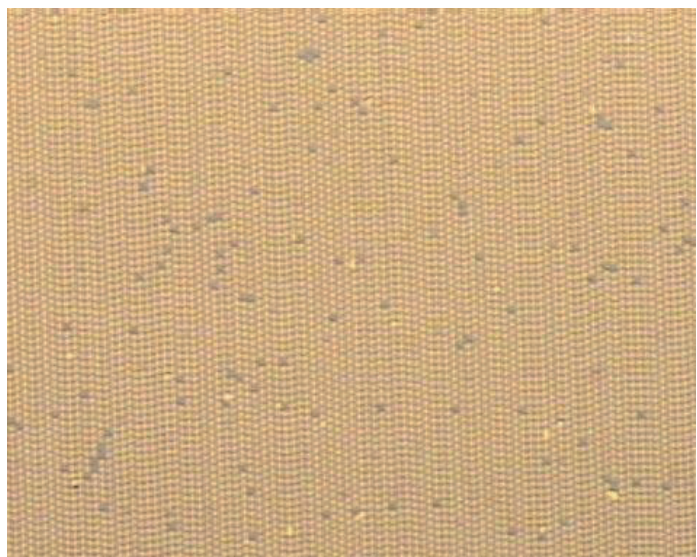

(a)

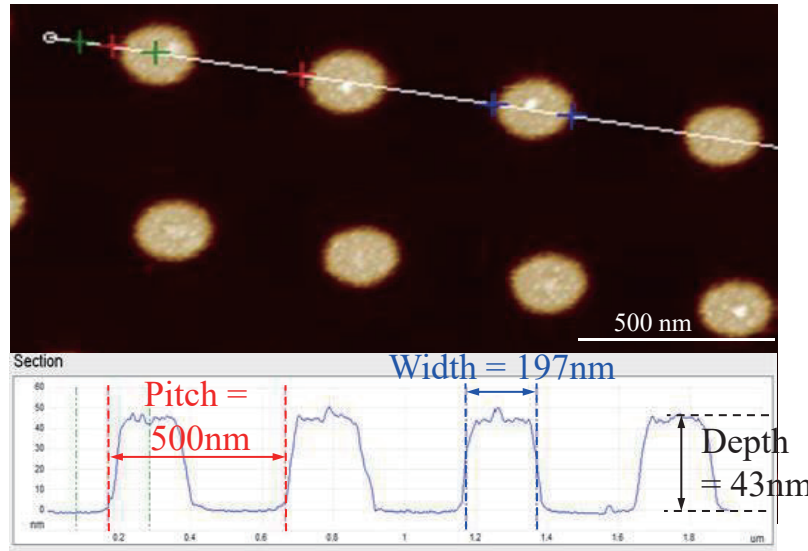

(b)

Fig. 3. (Color online) Topographical scan of the nanostructures using an (a) optical microscope and (b) AFM.

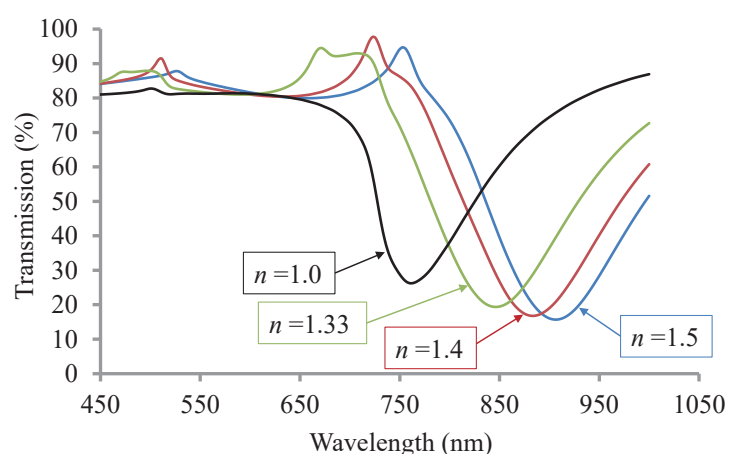

(a)

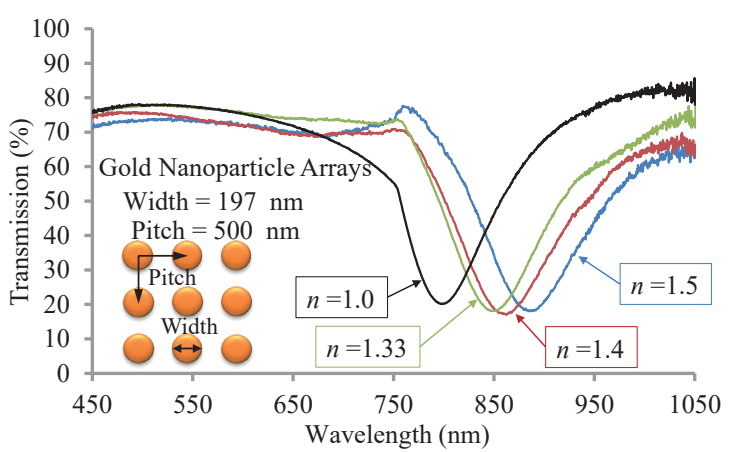

(b)

Fig. 4. (Color online) Transmission spectrum of the gold-nanoparticle-based biosensors in (a) FDTD simulation and (b) experiment. 


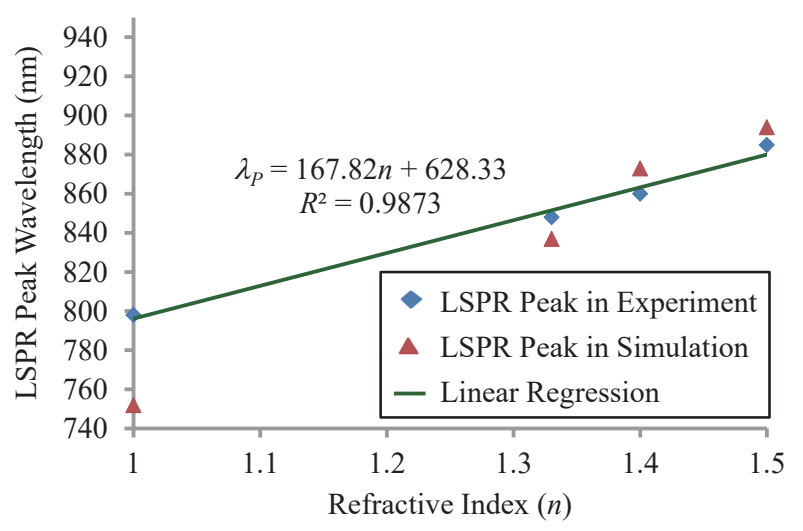

Fig. 5. (Color online) Measured and simulated transmission spectrum of the gold-nanoparticle-based biosensors with different refractive indexes.

\section{Conclusions}

In this study, a novel dual-wavelength laser DLDNS was developed to expose heatmode inorganic photoresist films and to efficiently fabricate LSPR biosensors, achieving manufacturing process abilities with high throughput (large area and quick production) and cost-effectiveness. Hence, the detection sensitivity of the LSPR biosensors with periodic arrays of nanostructures can be improved. The adjustments of the manufacturing process adopting DLDNS, to achieve gold-nanoparticle-based LSPR sensors for biosensor detection, include (1) power optimization of the laser diode drive controller, (2) composition of the heatmode inorganic photoresist films, and (3) regulation of the oxygen-plasma approach. The width, depth, and pitch of the fabricated periodic gold nanostructure arrays were 197, 43, and $500 \mathrm{~nm}$, respectively, and the measured transmission spectrum was $800 \mathrm{~nm}$. Therefore, these arrays are widely applicable to LSPR biosensors, and have the potential to be utilized in point-of-care diagnostics and food safety detection. Furthermore, the characteristics of the functional resonant wavelength/frequency and high sensitivity are suitable for applications in the biodetection and biomedical fields.

\section{Acknowledgments}

This work was supported by the Ministry of Science and Technology of Taiwan (R.O.C.) under Grant Nos. MOST 105-2221-E-019-027, 106-2221-E-019-052, and 106-2221-E-019-070.

\section{References}

1 J. Zhang, M. Irannejad, and B. Cui: Plasmonics 10 (2015) 831.

2 R. E. Ionescu, E. N. Aybeke, E. Bourillot, Y. Lacroute, E. Lesniewska, P. M. Adam, and J. L. Bijeon: Sensors 17 (2017) 236.

3 K. A. Willets and R. P. Van Duyne: Annu. Rev. Phys. Chem. 58 (2007) 267.

4 C. L. Haynes and R. P. Van Duyne: J. Phys. Chem. B 105 (2001) 5599. 
5 S. L. Zou, N. Janel, and G. C. Schatz: J. Chem. Phys. 120 (2004) 10871.

6 E. M. Larsson, J. Alegret, M. Käll, and D. S. Sutherland: Nano Lett. 7 (2007) 1256.

7 X. Lu, M. Rycenga, S. E. Skrabalak, B. Wiley, and Y. Xia: Annu. Rev. Phys. Chem. 60 (2009) 167.

8 C. F. Bohren and D. R. Huffman: Absorption and Scattering of Light by Small Particles (Wiley-VCH, New York, 1998) 2nd ed., Chap. 9.

9 P. Mulvaney: Langmuir 12 (1996) 788.

10 J. N. Anker, W. P. Hall, O. Lyandres, N. C. Shah, J. Zhao, and R. P. Van Duyne: Nat. Mater. 7 (2008) 442.

11 E. Petryayeva and U. J. Krull: Anal. Chim. Acta 706 (2011) 8.

\section{About the Authors}

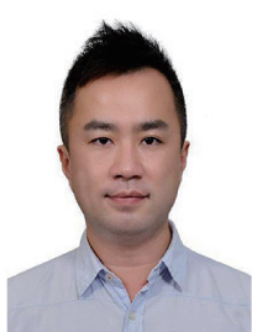

Hsing-Cheng Yu received his M.S. and Ph.D. degrees from the Department of Mechanical Engineering, National Chiao Tung University, Taiwan, in 2002 and 2010, respectively. From 2002 to 2011, he was a researcher at the Industrial Technology Research Institute, Taiwan. He is currently an associate professor in the Department of Systems Engineering and Naval Architecture, National Taiwan Ocean University, Taiwan. His research interests include biophotonics systems, sensor technology, optomechatronics system integration, actuator design and motor drive control, and ocean energy systems.

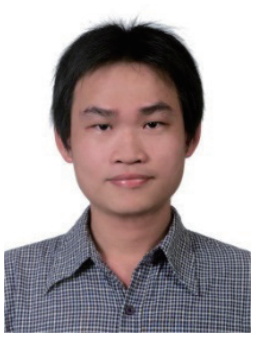

Shuen-Chen Chen received his M.S. degree from the Department of Systems Engineering and Naval Architecture, National Taiwan Ocean University. Since 2008, he has been a researcher at the Industrial Technology Research Institute, Taiwan. His research interests are in the fields of nano-optics, microstructure design, biochip technologies, electromechanical applications, and optical coherence tomography.

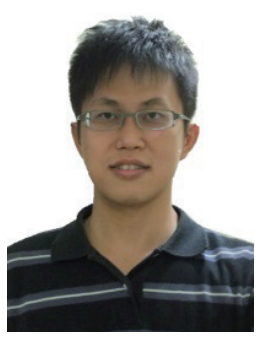

Ding-Zheng Lin received his B.S. degree from the Department of Mechanical Engineering, National Taiwan University, Taiwan, in 2001 and his M.S. and Ph.D. degrees from the Institute of Applied Mechanics, National Taiwan University, Taiwan, in 2003 and 2007, respectively. Since 2008, he has been a researcher at the Industrial Technology Research Institute, Taiwan. His research interests are in the fields of nano-optics, nanofabrication, plasmonic devices, optical biosensing, scanning near-field optical microscopy, and surface-enhanced Raman spectroscopy. 